\title{
LA CONSTRUCCIÓN SOSTENIBLE EN LATINOAMÉRICA
}

\author{
SUSTAINABLE ARCHITECTURE \\ AND CONSTRUCTION IN LATIN AMERICA
}

PERCY FLORES

Universidad de Lima
El presente artículo expone el estado actual y los avances que ha tenido la construcción sostenible en Latinoamérica; además, muestra diversas formas de contribuir a la sostenibilidad desde la arquitectura, ya sea desde criterios de diseño hasta investigaciones que aporten al tema. El objetivo es promover la importancia de una construcción sostenible, digna y eficiente para la sociedad, frente a los desafíos del cambio climático.

construcción sostenible, Latinoamérica, desarrollo sostenible, cambio climático
Recibido: 7 de julio del 2020

Aprobado: 2 de noviembre del 2020

doi: https://doi.org/10.26439/limaq2021.n007.5183

This article explains the current state and advances of sustainable construction in Latin America. In addition, it shows different ways to contribute to sustainability from an architectural perspective: from design criteria to research providing input to such topic. The objective of this research is to promote the importance of sustainable, dignified and efficient buildings for a society facing the challenges of climate change

sustainable construction, Latin America, sustainable development, climate change 


\section{INTRODUCCIÓN}

Para empezar a describir el panorama actual de la arquitectura sostenible en nuestro continente, es importante saber primero qué impacto está teniendo el efecto invernadero en América Latina y qué riesgo representa. El Quinto Informe de Evaluación del Grupo Intergubernamental de Expertos sobre el Cambio Climático (IPCC por sus siglas en inglés) concluye que, desde mediados del siglo xIX, el aumento de la temperatura de la superficie terrestre ha sido de $0,85^{\circ} \mathrm{C}$ y que, además, hay un crecimiento de las concentraciones de gases de efecto invernadero en la atmósfera; el informe considera a la actividad humana como la causa principal.

En América Latina los efectos negativos se pueden ver en el retroceso de los glaciares en los Andes, en el aumento de las precipitaciones, del riesgo de deslizamientos de tierra e inundaciones, erosión costera y una frecuencia mayor de eventos climáticos extremos (IPCC, 2014). Además, señala que los países en vías de desarrollo son más sensibles a sus efectos, pues su economía depende de sectores que son vulnerables al clima tales como la agricultura, los bosques y la energía hidroeléctrica. Por último, el informe sostiene que hay que tener en cuenta a los países que están en vías de desarrollo y que presenten una economía de lento crecimiento, pues serán menos capaces de afrontar los impactos que vendrán producto del efecto invernadero (IPCC, 2014).

Figura 1

Impactos del cambio climático en

América Latina

Fuente: IPCC

(2104)

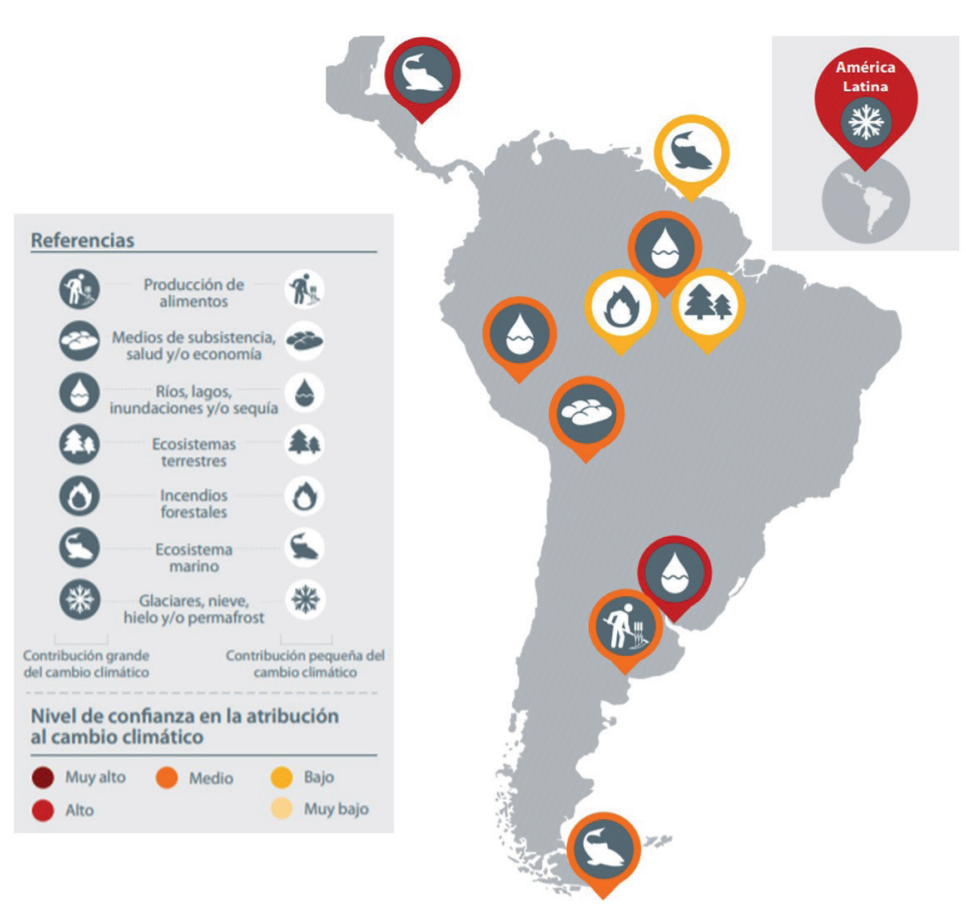




\begin{tabular}{|c|c|c|}
\hline . & $\begin{array}{l}\text { Nivel de } \\
\text { confianza }\end{array}$ & $\begin{array}{l}\text { Contribución al } \\
\text { cambio climático }\end{array}$ \\
\hline \multicolumn{3}{|l|}{ Nieve y hielo, ríos y lagos, inundaciones o sequía } \\
\hline Retroceso de los glaciares andinos & Alto & Grande \\
\hline $\begin{array}{l}\text { Cambios en los caudales extremos del río } \\
\text { Amazonas }\end{array}$ & Medio & Grande \\
\hline $\begin{array}{l}\text { Cambio de los patrones de descarga en los ríos en } \\
\text { la región occidental de los Andes }\end{array}$ & Medio & Grande \\
\hline \multicolumn{3}{|l|}{ Ecosistemas terrestres } \\
\hline $\begin{array}{l}\text { Aumento de mortalidad de los árboles y de los } \\
\text { incendios forestales en la Amazonía }\end{array}$ & Bajo & Pequeña \\
\hline $\begin{array}{l}\text { Degradación del bosque pluvial y recesión en la } \\
\text { Amazonía }\end{array}$ & Bajo & Pequeña \\
\hline \multicolumn{3}{|l|}{ Erosión costera y ecosistemas marinos } \\
\hline $\begin{array}{l}\text { Aumento de la decoloración de corales en la zona } \\
\text { occidental del Caribe }\end{array}$ & Alto & Grande \\
\hline $\begin{array}{l}\text { Degradación de manglares en las costas norte de } \\
\text { América del Sur }\end{array}$ & Bajo & Pequeña \\
\hline \multicolumn{3}{|l|}{ Producción de alimentos y medios de subsistencia } \\
\hline $\begin{array}{l}\text { Vulnerabilidad de las trayectorias de los medios de } \\
\text { subsistencia de los agricultores indígenas aimara }\end{array}$ & Medio & Grande \\
\hline $\begin{array}{l}\text { Aumento de los rendimientos agrícolas y expan- } \\
\text { sión de las zonas agrícolas en la zona suroriental } \\
\text { de América del Sur }\end{array}$ & Medio & Grande \\
\hline
\end{tabular}

Tabla 1

Impactos climáticos

observados en

América Latina

Fuente: IPCC

(2014)

Al saber las consecuencias que tendría el efecto invernadero, se implementaron políticas ambientales que buscan el equilibrio entre el desarrollo y el respeto por el medioambiente. Uno de los conceptos que apareció fue el de sostenibilidad, cuyo propósito aparece en 1987 dentro del informe de Brundtland: buscar satisfacer las necesidades del presente sin poner en riesgo los recursos de las generaciones futuras (ONU, 1987). Entonces, ¿cómo lograr un desarrollo sostenible desde la arquitectura y la construcción? Desde la etapa de diseño hasta el proceso de construcción se deben tener en cuenta varios puntos, entre los cuales destaca considerar la relación entre el contexto y la arquitectura, esto es pensar en un correcto emplazamiento y uso dentro de un territorio determinado y analizado para reducir su impacto ambiental; se debe tener en cuenta que las edificaciones sostenibles usan energía renovable para amortizar una parte de la energía que consumen a lo largo del día, dichas energías renovables pueden ser: solar, térmica, fotovoltaica, eólica, entre otras. Asimismo, se debe contemplar desde la etapa de diseño los materiales de construcción que se usarán, pues deben ser los más naturales y ecológicos posibles, priorizando siempre los de origen local para reducir el coste de su traslado, además del reciclaje y de la reutilización de los residuos al final de su 
ciclo de vida; por último, la vivienda debe responder a un diseño bioclimático óptimo y que garantice el confort térmico mediante sistemas pasivos de diseño (Ramírez, 2002).

\section{AVANCES EN ALGUNOS PAíSES DE AMÉRICA LATINA}

En cada país las iniciativas por mejorar fueron distintas, entre ellas se destacan las de Brasil, que ocupa el cuarto lugar en el mundo como país con mayor número de edificios con certificaciones LEED y que en el 2014 empezó a desarrollar una nueva certificación para construcciones residenciales y viviendas unifamiliares mediante un curso basado en leyes, condiciones climáticas y constructivas propias de los lugares en ese país (GBC Brasil, 2017). Argentina, cuyo gobierno, apoyado por el sector de construcción, desarrolló políticas públicas en favor de una construcción sostenible mediante el Ministerio de Ambiente y Desarrollo Sostenible, además, incorporó un manual de vivienda sustentable, producto de una colaboración interministerial, en donde se abordan temas desde el emplazamiento del proyecto hasta la construcción y su consumo energético, para brindar recomendaciones que ayuden a mejorar su desempeño ambiental (Gobierno de Argentina, 2019). Chile, que cuenta con un sistema de calificación energética de viviendas, promulgado por el Ministerio de Vivienda y Urbanismo, la cual funciona como herramienta voluntaria para evaluar la eficiencia energética de una nueva vivienda durante su etapa de uso. Con ello se pueden evaluar desde viviendas individuales hasta residencias y comparar el porcentaje de ahorro energético que tienen en comparación con una vivienda base (Ministerio de Vivienda y Urbanismo, y Ministerio de Energía, 2013).

Luego aparecen países con un menor desarrollo que los antes mencionados, estos son: Guatemala, que presenta el Plan Nacional de Desarrollo al 2032 como instrumento de planificación en donde aparecen cinco puntos de desarrollo nacional que fueron abordados y discutidos por más de 13000 personas durante 390 charlas realizadas en los municipios y departamentos; con ello se busca cumplir con desafíos sociales, económicos, culturales, ambientales y políticos para generar desarrollo a largo plazo (Secretaría de Planificación y Programación, 2014). También está el caso de Costa Rica, que con su norma RESET (requisitos para edificios sostenibles en el trópico) promueve el diseño y el agotamiento de los recursos como primera alternativa para sostenibilidad antes de recurrir a tecnologías, en donde se evalúan las etapas de diseño, construcción y la obra de una edificación según los criterios de la mencionada norma (Instituto de Normas Técnicas de Costa Rica, 2017), además, se imparten cursos de capacitación a arquitectos e ingenieros, quienes obtienen una licencia otorgada por el Colegio Federado de Ingenieros y Arquitectos. 


\begin{tabular}{ll}
\hline \multicolumn{1}{c}{ País } & \multicolumn{1}{c}{ Iniciativas } \\
\hline Brasil & $\begin{array}{l}\text { Cuarto país, según la US Green Building Council, con mayor número de edifi- } \\
\text { cios con certificaciones LEED. }\end{array}$ \\
Argentina & $\begin{array}{l}\text { En el } 2019 \text { se publica el Manual de Vivienda Sustentable. Aporta recomenda- } \\
\text { ciones para mejorar el desempeño ambiental de una construcción. }\end{array}$ \\
Chile & $\begin{array}{l}\text { En el } 2012 \text { aparece la Calificación Energética de Viviendas, la cual califica la } \\
\text { eficiencia energética de la vivienda en su etapa de uso. }\end{array}$ \\
Guatemala & $\begin{array}{l}\text { En el } 2014 \text { se publica el Plan Nacional de Desarrollo al } 2032 \text { como un instru- } \\
\text { mento de planificación. }\end{array}$ \\
Costa Rica & $\begin{array}{l}\text { Cuenta con la norma RESET, cuyo objetivo es priorizar la capacidad de } \\
\text { diseño para soluciones bioclimáticas en lugar del uso de tecnologías. }\end{array}$
\end{tabular}

Sin embargo, a pesar del avance que se ha visto en los últimos años, el desarrollo en algunos países de Latinoamérica sigue siendo básico. En México, mediante un informe, la arquitecta Alejandra Dueñas del Río narra la falta de interés para desarrollar arquitectura sustentable; expone la falta de atención que tiene el gobierno para hacer que se cumplan los lineamientos, pues a pesar de tener reglamentos nacionales que regulan y promueven dentro de un marco legal la sostenibilidad, como la Estrategia Nacional para la Vivienda Sustentable, promulgada en el 2013, o La Ley de Vivienda, promulgada en el 2006, no se sanciona o se incentiva al constructor para llevar a cabo una edificación sustentable integral; también está la falta de un sistema de calificación y certificación adecuado para los sistemas constructivos que se desarrollan dentro de México, ya que en diversos lugares del interior los materiales y la forma de construir cambia (Dueñas del Río, 2013). En Colombia también existen documentos normativos relacionados que apoyan la construcción sostenible, como el Macroproyecto de Vivienda de Interés Social y Prioritario, que está vigente desde el 2010 y en el cual se propone el uso de materiales térmicos, iluminación eficiente y uso de electrodomésticos oficiales, el cual es efectuado mediante el Gobierno Nacional a través de su Ministerio de Energía y Minas, el Ministerio de Ambiente y Desarrollo Sostenible, así como del Ministerio de Vivienda, Ciudad y Territorio y de las instituciones privadas. Sin embargo, solo se han limitado a la emisión de normativas y regulaciones, mas no a mecanismos de supervisión que permitan corroborar y controlar de manera eficaz el cumplimiento de las medidas de ahorro, como también pasa en México, por lo que queda a decisión del constructor si desea aplicar prácticas de sostenibilidad en sus proyectos constructivos (Rodríguez, Villadiego, Padilla y Osorio, 2018).

Otro caso es el de Chile, en donde también se han promovido normativas de mejora en relación con la sostenibilidad en construcción, teniendo éxito en proyectos públicos y privados, y posicionando a ese país como referente en comparación con los países de la región; sin embargo, este crecimiento no contó con una política pública adaptada a una normativa local, es por esto que gran parte de las infraestructuras se construyeron bajo estándares de países con condiciones políticas, sociales y geográficas diferentes, detalla Alexis Núñez, jefe de línea de Desarrollo de Edificación de la Agencia
Tabla 2

Avances en

América Latina

Fuente: GBC Brasil

(2017); Gobierno

de Argentina

(2019); Ministerio

de Vivienda

y Urbanismo,

Ministerio de

Energía (2013);

Secretaría de

Planificación y

Programación

(2014); Instituto de

Normas Técnicas

de Costa Rica

(2017) 
Tabla 3

¿Qué es lo que aún

falta en algunos

países?

Fuente: Dueñas

del Río (2013);

Rodríguez,

Villadiego, Padilla,

y Osorio (2018);

Revista EMB

Construcción

(2016); Martins

(2016); Ministerio

de Industria,

Energía y Minería

(2015); Cubillos

González (2012)
Chilena de Eficiencia Energética; demostrando así que hay edificaciones que cumplen con diversos criterios de sustentabilidad, pero que están separados del contexto urbano (Construcción, 2016). Por otro lado, Uruguay se está posicionando como líder en inversión para la utilización de energía eólica; además, aparece como el tercer país en el mundo y el primero en Latinoamérica con mayor porcentaje de este tipo de energía, pues el $22 \%$ de su electricidad es generada con el viento (Martins, 2016); sin embargo, su capital, Montevideo aún está lejos de ser considerada una "ciudad verde", debido a que no tienen muchas iniciativas de construcción y eficiencia energética. De las pocas que tienen destaca el Proyecto de Eficiencia Energética, el cual es un programa de alcance nacional que está orientado a promover un uso eficiente de todos los tipos de energía en todos los sectores económicos, también muestra consejos y herramientas para aprovechar al máximo la energía de las casas y nombra a empresas de servicios energéticos que pueden funcionar como proveedores de equipamiento eficiente (Ministerio de Industria, Energía y Minería, 2015). En Colombia, el investigador Rolando Cubillos expone la falta de interés del sector construcción en el campo de la sostenibilidad, pues las intervenciones que han hecho responden más a la presión de normas y acuerdos internacionales en lugar de un compromiso propio, también sostiene que en Bogotá se requiere que la empresa pública y privada se interese en el tema; además señala la falta de una estrategia que permita construir una política clara que defina conceptos propios del desarrollo sostenible y afirma que, con la definición y aplicación de estos conceptos, se construirán estrategias que permitan que la ciudad sea direccionada hacia la sostenibilidad (Cubillos González, 2012).

\begin{tabular}{|c|c|}
\hline País & ¿Qué es lo que falta? \\
\hline México & $\begin{array}{l}\text { Falta de atención para que se cumplan los reglamentos nacionales que } \\
\text { promuevan y regulen la sostenibilidad. No se sanciona o se incentiva al } \\
\text { constructor para llevar a cabo una edificación sustentable integral. }\end{array}$ \\
\hline Colombia & $\begin{array}{l}\text { A pesar de tener documentos normativos que apoyan la construcción } \\
\text { sostenible, no hay mecanismos de supervisión que permitan controlar de } \\
\text { manera eficaz el cumplimiento de las medidas de ahorro. }\end{array}$ \\
\hline Chile & $\begin{array}{l}\text { Cuenta con una política pública que no está adaptada a una normativa } \\
\text { local. Se construye bajo estándares de países con condiciones políticas, } \\
\text { sociales y geográficas diferentes. }\end{array}$ \\
\hline Uruguay & $\begin{array}{l}\text { Faltan más iniciativas de políticas públicas. Entre las existentes destaca } \\
\text { la Política Energética } 2005-2030 \text { propuesta por el Ministerio de Industria, } \\
\text { Energía y Minería. }\end{array}$ \\
\hline Colombia & $\begin{array}{l}\text { Falta de interés del sector construcción, pues las intervenciones responden } \\
\text { más a la presión de normas en lugar de un compromiso propio. En Bogotá } \\
\text { falta una estrategia que permita una política clara. }\end{array}$ \\
\hline
\end{tabular}

PROPUESTAS PARA SEGUIR CON EL CAMBIO

Con los ejemplos mencionados, se puede observar que en algunos países los factores que impiden un desarrollo sostenible se repiten. Dichos factores son la falta de normativas por parte del Estado que apoyen la eficiencia energética 
y la construcción sostenible y que, como punto más importante, supervise que los encargados de realizar los proyectos cumplan con estas normas; el no promover una certificación sostenible en edificaciones; la falta de incentivos financieros y la poca formación profesional y técnica. Además, en comparación con países europeos, más desarrollados e industrializados - entre los cuales destacan Dinamarca, Alemania, Holanda, Austria, Suecia y Suizaquienes han desarrollado estudios de impacto ambiental efectivos, análisis de ciclo de vida (ACV) y el reciclaje de materiales (Gómez, 2008), así como una fuerte participación gubernamental, de instituciones universitarias y empresas privadas; la construcción sostenible en Latinoamérica no ha avanzado al mismo ritmo. Un ejemplo son las escasas iniciativas de investigación, desarrollo e innovación $(\mathrm{I}+\mathrm{D}+\mathrm{i})$. En cuanto al sector construcción, se han limitado al desarrollo de soluciones puntuales, tales como cerramientos, tabiquería liviana, aislantes térmicos, entre otros; dejando de lado propuestas más trascendentes como son las de tipo estructural.

Bajo este panorama, han aparecido iniciativas y propuestas para concientizar a la sociedad y a los arquitectos sobre el cuidado medioambiental desde el enfoque de la arquitectura. Pedro Montilla Moreno (2012), en un artículo escrito para la Universidad de los Andes en Venezuela, propone las siguientes estrategias de acción: empezar con la creación y desarrollo de normativas oficiales medioambientales de parte de un Estado promotor de viviendas sostenibles; investigar, incentivar el desarrollo e innovación orientado al uso de tecnologías y recursos locales disponibles; implementar estudios de análisis del ciclo de vida (ACV) de los materiales que forman parte del proceso constructivo, así como evaluar constantemente, calificar y otorgar las certificaciones energéticas correspondientes a las edificaciones (Montilla Moreno, 2012).

Otras estrategias de acción se muestran en la obra colectiva dirigida por Sylvie Nail y publicada en el 2016. Dicho trabajo multidisciplinario muestra diversas maneras de abordar el desafío del cambio climático en América Latina, ya sea desde políticas gubernamentales hasta el desarrollo de estrategias más críticas. El trabajo empieza mostrando diferentes ciudades en múltiples escalas para saber qué medidas tomar a corto y largo plazo, junto a los elementos más importantes que es necesario tomar en cuenta en la gestión de las problemáticas relacionadas con el cambio climático. En esta obra es importante poner de relieve las propuestas que pueden ser aplicadas desde la arquitectura. Los escritores que destacan son: a) A. Lampis que propone pensar "fuera de la caja", es decir, tener en cuenta ciertos criterios para pensar políticas públicas, las cuales, además del conocimiento de los hechos científicos, deberían considerar una argumentación más adaptada a la cultura y al contexto local para que no sea percibida como un elemento aislado con su entorno (Universidad Externado de Colombia, 2016, p. 107); b) A. Fry, quien sostiene que el cambio climático obliga a mirar a las ciudades de una manera diferente, pues se tiene que tener en cuenta la dimensión del tiempo y la dimensión socioespacial del crecimiento urbano actual; además, enfatiza en la necesidad de corregir la desigualdad socioespacial ya que deja expuestos a los más vulnerables a las consecuencias del cambio climático (Universidad Externado de Colombia, 2016, p. 199); c) L. Katzchner, que expone 
Tabla 4

Propuestas de diversos autores

Fuente: Montilla Moreno (2012) que las consideraciones climáticas, por el hecho de que estas no son tomadas en cuenta, tienen poco impacto en la planificación urbana; además, sostiene que los aportes de la climatología, junto al diseño urbano, deberían resultar en un trabajo interdisciplinario; también presenta el mapa climático urbano (Urban Climatic Map) como una herramienta para tomar decisiones, desde la planificación, y mejorar el confort térmico a diferentes escalas. Muestra ejemplos en Kassel y Freiburg, en Alemania, y en Belo Horizonte y Salvador de Bahia, en Brasil (Universidad Externado de Colombia, 2016, p. 425); d) G. Penagos, que presenta su propuesta mostrando el ejemplo en Medellín (Colombia) de una formulación de política de construcción sostenible tomando en cuenta la mitigación y adaptación al cambio climático; además, muestra datos en donde expone que el $40 \%$ de las emisiones de gases de efecto invernadero las produce el sector construcción, por lo que resulta necesario calcular las emisiones del ciclo de vida de las construcciones, desde los materiales hasta los servicios que va a necesitar para tener políticas que ayuden a mejorar la eficiencia (Universidad Externado de Colombia, 2016, p. 519). Al final de la obra, S. Nail concluye argumentando que el conocimiento científico existe y que estos deberían abordarse a largo plazo dentro de una agenda climática. Resalta la necesidad de influenciar en la ciudadanía para que exija a sus dirigentes tomar una acción inmediata que sea coherente e inclusiva, además de abordar un cambio social y cultural en donde seamos conscientes de las consecuencias medioambientales que estamos afrontando (Universidad Externado de Colombia, 2016).

\begin{tabular}{|c|c|}
\hline \multicolumn{2}{|r|}{ Propuestas para seguir con el cambio } \\
\hline Autor & Iniciativa \\
\hline \multirow[t]{3}{*}{ Pedro Montilla } & $\begin{array}{l}\text { Creación y desarrollo de normativas oficiales medioambientales de parte } \\
\text { de un Estado promotor de viviendas sostenibles. }\end{array}$ \\
\hline & $\begin{array}{l}\text { Incentivar la investigación, desarrollo e innovación orientados al uso de } \\
\text { tecnologías y recursos locales disponibles. }\end{array}$ \\
\hline & $\begin{array}{l}\text { Implementar estudios de análisis de ciclo de vida (ACV) de materiales de } \\
\text { construcción. }\end{array}$ \\
\hline A. Lampis & $\begin{array}{l}\text { Tener en cuenta ciertos criterios, adaptados a la cultura y al contexto local, } \\
\text { al momento de pensar en políticas públicas. }\end{array}$ \\
\hline \multirow[t]{2}{*}{ A. Fry } & $\begin{array}{l}\text { Mirar a las ciudades de una manera diferente: considerar la dimensión } \\
\text { del tiempo y la dimensión socioespacial del crecimiento urbano actual. }\end{array}$ \\
\hline & $\begin{array}{l}\text { Enfatiza en la necesidad de corregir la desigualdad socioespacial, ya que } \\
\text { expone a los más vulnerables de las consecuencias del cambio climático. }\end{array}$ \\
\hline \multirow[t]{2}{*}{ L. Katzchner } & $\begin{array}{l}\text { Considerar las consideraciones climáticas al momento de la planificación } \\
\text { urbana. }\end{array}$ \\
\hline & $\begin{array}{l}\text { Propone un trabajo interdisciplinario entre los aportes de la climatología } \\
\text { y el diseño urbano para mejorar el confort térmico en diferentes escalas. }\end{array}$ \\
\hline \multirow[t]{2}{*}{ G. Penagos } & $\begin{array}{l}\text { Con el ejemplo de Medellín, propone una formulación de política de } \\
\text { construcción sostenible que tome en cuenta la mitigación y adaptación } \\
\text { al cambio climático. }\end{array}$ \\
\hline & $\begin{array}{l}\text { Calcular las emisiones de ciclo de vida de las construcciones para tener } \\
\text { políticas que ayuden a mejorar la eficiencia. }\end{array}$ \\
\hline S. Nail & $\begin{array}{l}\text { Con el conocimiento científico acumulado se debería abordar a largo } \\
\text { plazo una agenda climática. }\end{array}$ \\
\hline
\end{tabular}




\section{¿Y QUÉ PASA EN EL PERÚ?}

A diferencia de nuestros países vecinos, el caso del Perú es particular. A pesar de generar el 0,4\% de los gases de efecto invernadero (PNUD, 2014), según un informe publicado en el 2004 por la Universidad de Manchester, Perú es el tercer país más vulnerable del mundo al cambio climático. Los investigadores climáticos sostienen que, al verse afectadas las principales fuentes de agua, que son los glaciares, Lima entraría en una gran sequía y aparecerían fenómenos hidrometeorológicos, como fuertes lluvias, inundaciones, heladas, entre otros; además, también consideremos el problema de que el $90 \%$ de la población vive en zonas áridas, semiáridas y subhúmedas, en las que, según el informe, en los próximos 40 años el Perú poseerá solo el $60 \%$ del agua potable de la que dispone ahora (Tarabochia, 2014).

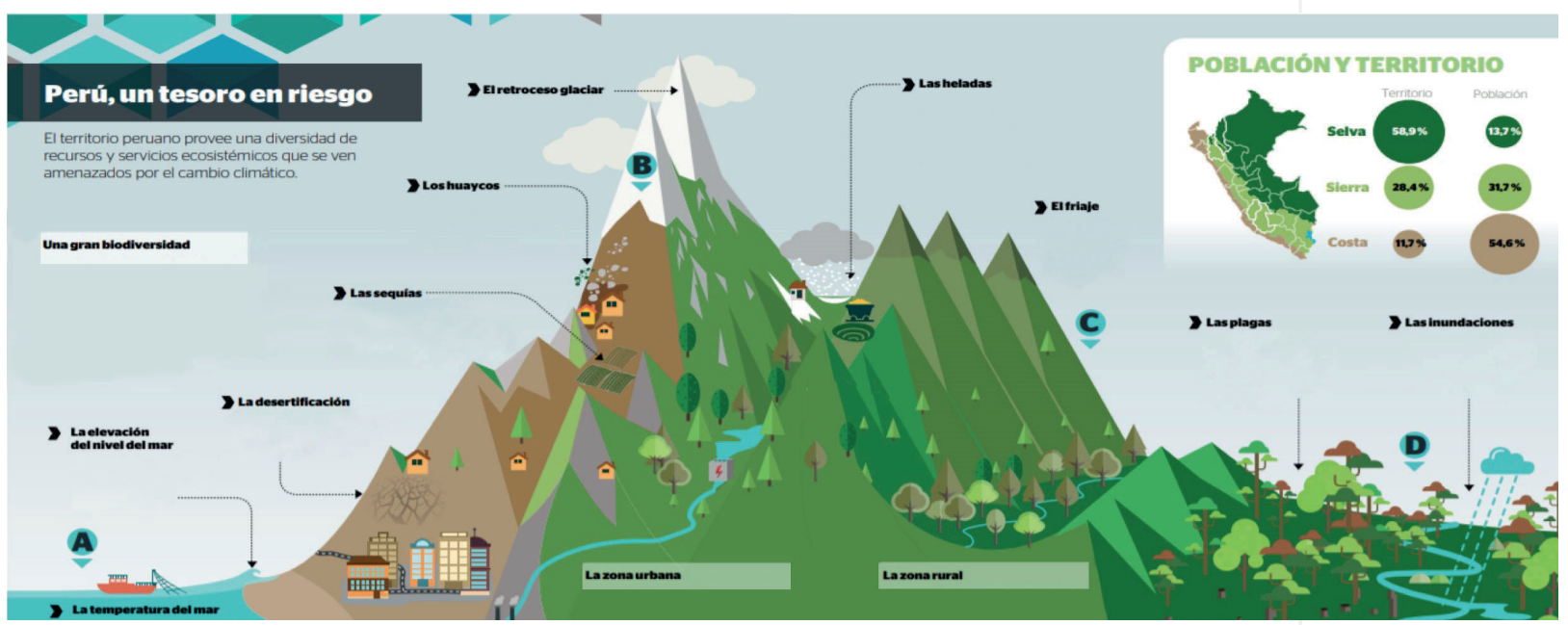

Con el riesgo que representa el cambio climático, ¿qué se hace para tener un desarrollo sostenible desde la arquitectura y construcción? En cuanto a medidas y acciones para migrar a un modelo de construcción sostenible, se tiene el informe propuesto por el equipo "Foro Ciudades para la Vida", que en colaboración con el Ministerio de Vivienda, Construcción y Saneamiento, propone cuatro estrategias de acción, junto con sus respectivas medidas por cada punto, las cuales son: modificar las reglas de la gestión de la construcción, la cual impulsa al MVCS a establecer alianzas estratégicas con organismos privados, sociedad civil y entidades públicas, con el fin de que los agentes público y privados adopten medidas amigables con el ambiente; estimular la oferta de procesos constructivos sostenibles para la construcción, motivando a los principales distribuidores de productos a abastecerse con productos y tecnologías amigables con el ambiente; estimular la demanda de procesos constructivos sostenibles para la construcción, con el fin de que los usuarios tengan mayor conciencia del rol que cumplen en un contexto de desarrollo
Figura 2.

Amenazas del cambio climático en el Perú

Fuente: Ministerio del Ambiente (2016), editado por el autor 
sostenible, demandando así edificaciones que se adapten a las condiciones climáticas que presenta su región; y generar capacidades, conocimientos e información para la adaptación (Miranda, Neira, Torres y Valdivia, 2014). En cuanto a informes que promuevan estrategias de diseño ecoamigables, está el que escribieron en el 2005 los arquitectos Rayter, Fuster y Zúñiga, en el cual proponen un "mapa climático para diseño arquitectónico". En él se clasificaba al Perú en zonas conforme a variables climáticas - temperatura, precipitaciones, vientos, sol, etcétera-. Dicho análisis coincide en muchos rasgos con el altitudinal de las "ocho regiones naturales" de Javier Pulgar Vidal, formulada en 1938, que incorporaba el clima, la acción trópica, vegetación y fauna (Yarasca, 2019). Considerando el mapa climático, el arquitecto Martín Wieser propone una serie de recomendaciones de diseño que están ligadas a la calidad ambiental interior, desde el punto de vista térmico, teniendo en cuenta los diferentes climas que tiene el Perú. Wieser considera que en el proceso de diseño arquitectónico no se tiene mucho interés en las consideraciones climáticas; además de que hay poca información, adaptada a nuestro país, vinculada a ofrecer confort térmico a los usuarios con criterios de eficiencia energética (Wieser, 2011).

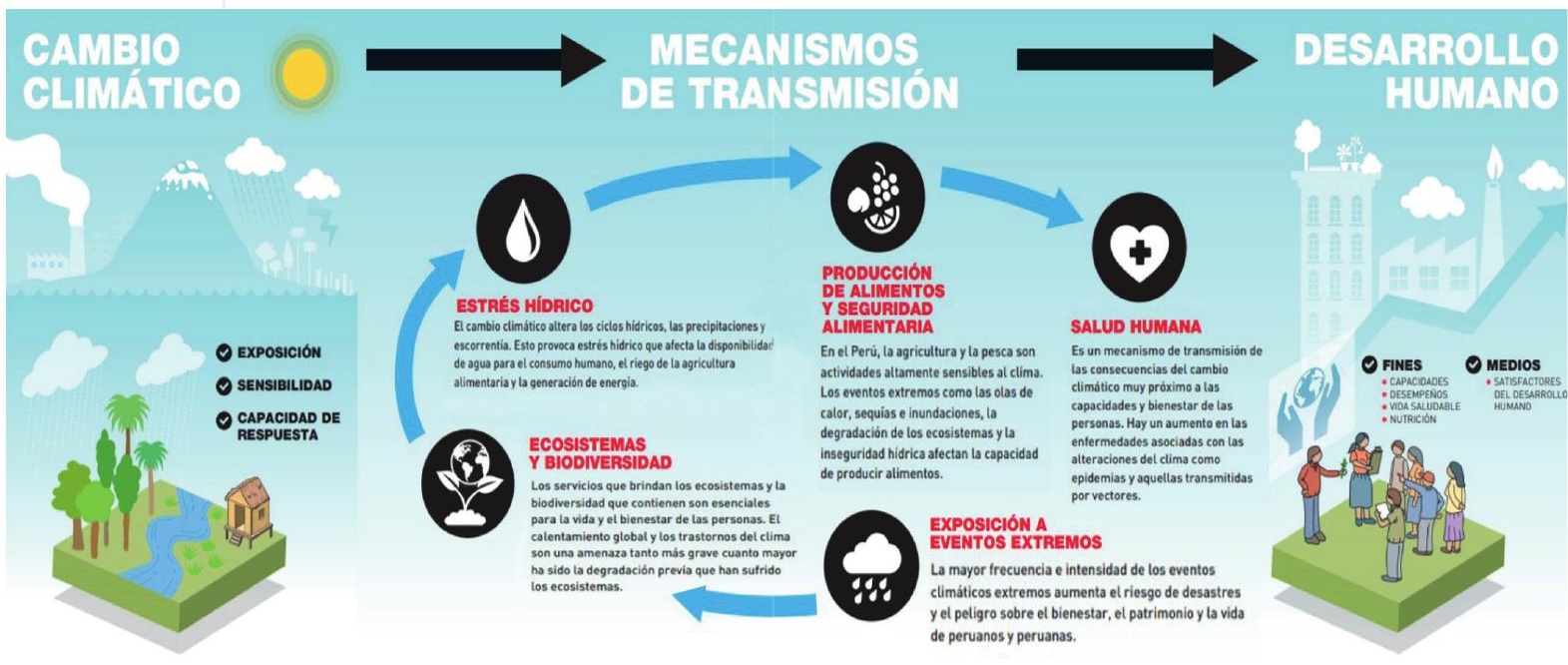

Figura 3. Cambio climático y desarrollo humano

Fuente: PNUD (2014), editado por el autor
En cuanto a normas y políticas tenemos al Ministerio de Vivienda, Construcción y Saneamiento, que mediante el Plan Nacional de Gestión Ambiental 20112021, establece mecanismos de fiscalización y control ambiental de las actividades ligadas a la construcción, urbanismo y saneamiento (Ministerio del Ambiente, Plan de Acción Ambiental Perú 2011-2021, 2011). Junto con el ministerio, la Dirección Nacional de Construcción (DNC) también se encarga de aprobar los estudios de impacto ambiental y expedir certificaciones ambientales. La DNC también está a cargo de más de quince comités técnicos especializados en el desarrollo de normas técnicas edificatorias, algunas son: de uso de energías renovables, de acondicionamiento térmico y lumínico con eficiencia energética, de diseño y construcción de ciclovías. Asimismo, han 
desarrollado estudios para promover soluciones constructivas en zonas de heladas en Huancavelica en el 2012 y para zonas de calor, como Iquitos, Ica y Piura, en el 2014 (Miranda, Neira, Torres y Valdivia, 2014).

A pesar de que en la última década han aparecido distintas iniciativas del Estado para hacer frente al cambio climático, es importante tener conciencia de que aún nos encontramos en una etapa inicial en cuanto a la incorporación de medidas de eficiencia energética; reconozcamos que aún con las políticas gubernamentales, del sector privado y de la sociedad civil, todavía no se incorpora el enfoque de desarrollo sostenible. El cambio será exitoso si se reconoce la poca conciencia ambiental que hay entre profesionales ligados a la construcción, que aún usan procesos y técnicas convencionales que son poco sostenibles. En lugar de considerar el conocimiento ancestral de materiales y técnicas propias del lugar, se debe considerar que tanto la tradición como la modernidad deberían complementarse para un desarrollo sostenible.

\section{CONCLUSIÓN}

El desarrollo sostenible debe asumirse como uno de los ejes principales para reducir el impacto que tendrá el efecto invernadero. Es importante considerar e impulsar la investigación, el desarrollo y la innovación $(\mathrm{I}+\mathrm{D}+\mathrm{i})$ de materiales y técnicas constructivas tradicionales y propias de cada lugar en donde se trabaja, junto al análisis del ciclo de vida (ACV) de los materiales que se usarán. Sus resultados serán de utilidad estratégica y decisiva para la toma de decisiones frente a un proyecto constructivo, en cualquier entorno y situación. Es necesario seguir con políticas del Estado que promuevan y controlen el correcto desarrollo de una construcción que cause el mínimo impacto ambiental. Tengamos en cuenta que el agravamiento del cambio climático es inevitable en las próximas décadas; sigamos entonces, desde nuestro lado, generando conciencia para enfrentar juntos los desafíos que plantea el cambio climático.

\section{REFERENCIAS}

Álvarez, A. M. (2013). (Des) Igualdad socio espacial y justicia espacial: nociones clave para una lectura crítica de la ciudad. Polis, 12(36), 265-287.

Cedillos, O. A., y Lara, O. B. (2009). La arquitectura sostenible en la formación del arquitecto (trabajo de graduación previo al grado de arquitecto). Universidad de El Salvador, San Salvador, El Salvador.

Cubillos González, R. A. (2012). La tecnología sostenible aplicada al proyecto arquitectónico. Bogotá: Universidad Católica de Colombia.

Dueñas del Río, A. (2013). Reflexiones sobre la arquitectura sustentable en México. Revista Legado de Arquitectura y Diseño, (14), 77-91. 
Estrada, D. (Ed.). (6 de noviembre del 2008). Inter Press Service Agencia de Noticias. Recuperado de http://www.ipsnoticias.net/2008/11/arquitectura -america-latina-utopia-sustentable/

GBC Brasil. (16 de octubre del 2017). Certificação para o setor residencial contempla quesitos sociais. Recuperado de https://www.gbcbrasil.org.br/ certificacao-para-o-setor-residencial-contempla-quesitos-sociais/

Gobierno de Argentina. (2019). Manual de Vivienda Sustentable. Buenos Aires. Recuperado de https://www.argentina.gob.ar/ambiente/desarrollo-soste nible/vivienda/manual

Gómez, J. (2008). Ecodiseño y Ecoeficiencia en la Industria. Retos y Oportunidades. Zaragoza: Instituto Tecnológico de Aragón. Recuperado de https://issuu. com/ninamoya/docs/compilaci_n_ecodise_o

Instituto de Normas Técnicas de Costa Rica. (2017). Construcción RESET. Requisitos para Edificaciones Sostenibles en el Trópico. Costa Rica: INTECO.

Martins, A. (14 de marzo del 2016). Cómo Uruguay logró ser el país con mayor porcentaje de energía eólica de América Latina. BBC Mundo. Recuperado de https://www.bbc.com/mundo/noticias/2016/03/160314_uru guay_energia_eolica_am

Máxima eficiencia. (2016). EMB Construcción. Recuperado de http://www.emb.cl /construccion/articulo.mvc?xid=3671\&edi=169\&xit=construccion-susten table-en-chile-maxima-eficiencia

MDUyT. (2014). Buenos Aires, ciudad verde: una mirada prospectiva al paisaje urbano 2014. Buenos Aires: Ministerio de Desarrollo Urbano y Transporte de la Ciudad de Buenos Aires.

Ministerio de Industria, Energía y Minería. (2015). Plan Nacional de Eficiencia Energética 2015-2024. Ministerio de Industria, Energía y Minería, Montevideo. Recuperado de https://smart-lighting.es/wp-content/uploads/2015/08/MlUruguay-Plan-Nacional-de-Eficiencia-Energetica2015-2024.pdf

Ministerio de Vivienda y Urbanismo, Ministerio de Energía. (2013). Calificación Energética de Viviendas en Chile. Santiago: Ministerio de Vivienda y Urbanismo.

Ministerio del Ambiente. (2011). Plan de Acción Ambiental Perú 2011-2021. Lima.

Ministerio del Ambiente. (2016). El Perú y el cambio climático. Tercera comunicación nacional del Perú a la Convención Marco de las Naciones Unidas sobre Cambio Climático. Lima.

Miranda, L., Neira, E., Torres, R., y Valdivia, R. (2014). Perú hacia la construcción sostenible en escenarios de cambio climático. Lima: Ministerio de Vivienda, Construcción y Saneamiento. Recuperado de http://www.cies.org.pe/ sites/default/files/investigaciones/edicion_final_estudio_construccion_ sostenible.pdf 
Montilla Moreno, P. (2012). La construcción de edificaciones sostenibles. Perspectivas, estrategias y retos en Latinoamérica. Mérida: Universidad de Los Andes.

Nail, S. (Ed.). (2016). Cambio climático. Lecciones de y para ciudades de América Latina. Bogotá: Universidad Externado de Colombia.

Organización de las Naciones Unidas. (1987). Informe Brundtland. Oxford University Press. Recuperado de http://www.ecominga.uqam.ca/PDF/BIBLIOGRA PHIE/GUIDE_LECTURE_1/CMMAD-Informe-Comision-Brundtland-sobreMedio-Ambiente-Desarrollo.pdf

Panel Intergubernamental del Cambio Climático. (2014). Quinto Informe de Evaluación del Panel Intergubernamental del Cambio Climático. IPCC. Ginebra: IPCC. Recuperado de ipcc.ch/site/assets/uploads/2018/02/SYR AR5_FINAL_full_es.pdf

Programa de las Naciones Unidas para el Desarrollo. (2014). Cambio climático y territorio. En busca de sostenibilidad para el desarrollo humano en el Perú. Lima: Biblioteca Nacional del Perú. Recuperado de https:// www.pe.undp.org/content/peru/es/home/library/poverty/lnformesobre desarrollohumano2013//DHPeru2013.html

Ramírez, A. (2002). La construcción sostenible. Física y Sociedad (13), 54. Recuperado de http://www.cofis.es/pdf/fys/fys13/fys13_completo.pdf

Revista Construcción EMB. (Noviembre del 2016). Construcción sustentable en Chile.

Rodríguez, L., Villadiego, K., Padilla, S., y Osorio, H. (2018). Arquitectura y urbanismo sostenible en Colombia. Una mirada al marco reglamentario. Bitácora Urbano Territorial, 26.

Secretaría de Planificación y Programación. (2014). Plan Nacional de Desarrollo K'atun: nuestra Guatemala 2032. Ciudad de Guatemala: Segeplán.

Tarabochia, M. L. (17 de enero del 2014). Perú es el tercer país más vulnerable del mundo al cambio climático. SERVINDI. Recuperado de https://www. servindi.org/actualidad/99300

Wieser, M. (2011). Consideraciones bioclimáticas en el diseño arquitectónico: el caso peruano. Lima: PUCP, Departamento de Arquitectura.

Yarasca, C. (2019). El problema de la distancia. Las plataformas multisectoriales "tambos" como una oportunidad proyectual sistemática para el territorio rural peruano. Textos de Investigación (11), 150-165. 